\title{
ON THE POSSIBILITY \\ OF DIFFERENTIATING TERM BY TERM THE DEVELOPMENTS \\ FOR AN ARBITRARY FUNCTION OF ONE REAL VARIABLE \\ IN TERMS OF BESSEL FUNCTIONS*
}

BY

WALTER B. FORD

1. The developments for an arbitrary function $f(x)$ of the real variable $x$ in terms of BEsser's function $J_{\nu}(x)$ ( $\nu$ real) may be classed into three general divisions as follows:

I.

$$
\sum_{1}^{\infty} q_{n} J_{\nu}\left(\lambda_{n} x\right)
$$

where

$$
q_{n}=\frac{2}{J_{\nu}^{\prime 2}\left(\lambda_{n}\right)} \int_{0}^{1} x f(x) J_{\nu}\left(\lambda_{n} x\right) d x
$$

$\lambda_{n}$ being one of the positive roots of the transcendental equation $J_{\nu}(x)=0$.

where

$$
(2 \nu+2) \int_{0}^{1} f(x) x^{\nu+1} d x+\sum_{1}^{\infty} q_{n}^{\prime} J_{\nu}\left(\lambda_{n}^{\prime} x\right)
$$

$$
q_{n}^{\prime}=\frac{2}{J_{\nu}^{2}\left(\lambda_{n}^{\prime}\right)} \int_{0}^{1} x f(x) J_{\nu}\left(\lambda_{n}^{\prime} x\right) d x,
$$

$\lambda_{n}^{\prime}$ being one of the positive roots of the transcendental equation

$$
x J_{\nu}^{\prime}(x)-\nu J_{\nu}(x)=0 .
$$

III.

$$
\sum_{1}^{\infty} q_{n}^{\prime \prime} J_{\nu}\left(\lambda_{n}^{\prime \prime} x\right)
$$

* Presented to the Society September 3, 1902. Received for publication September 30, 1902. 
where

$$
q_{n}^{\prime \prime}=\frac{2 \lambda_{n}^{\prime \prime 2}}{\left\{h(2 \nu+h)+\lambda_{n}^{\prime \prime 2}\right\} J_{\nu}^{2}\left(\lambda_{n}^{\prime \prime}\right)} \int_{0}^{1} x f(x) J_{\nu}\left(\lambda_{n}^{\prime \prime} x\right) d x,
$$

$\lambda_{n}^{\prime \prime}$ being one of the positive roots of the transcendental equation

$$
x J_{\nu}^{\prime}(x)-(h+\nu) J_{\nu}(x)=0 \quad(h \text { real and } \neq 0) .
$$

With reference to these three developments it is our present purpose to determine a set of sufficient conditions for $f(x)$ under which the series obtained by differentiating series I or II term by term will converge to the limit $f^{\prime}(x)$. The discussion naturally presupposes some facts concerning the convergence of the series in question, or of some related series, and thus we shall begin by stating the following established results:*

"If

$$
\begin{aligned}
P_{\nu}(x)=\frac{J_{\nu}(x)}{x^{\nu}}=\frac{1}{2^{\nu} \Gamma(\nu+1)}\left\{1-\frac{x^{2}}{2(2 \nu+2)}\right. & \\
& \left.\quad+\frac{x^{4}}{2 \cdot 4(2 \nu+2)(2 \nu+4)}-\cdots\right\}
\end{aligned}
$$

and if $f(x)$ is an arbitrary function of the real variable $x$ defined throughout the interval $0 \leqq x \leqq 1$ we shall have for any special value of $x$ within an. interval $\left(a^{\prime}, b^{\prime}\right)\left(0<a^{\prime}<b^{\prime}<1\right)$

where

$$
f(x)=\sum_{1}^{\infty} p_{n} P_{\nu}\left(\lambda_{n} x\right)
$$

$$
p_{n}=\frac{2}{P_{\nu}^{\prime 2}\left(\lambda_{n}\right)} \int_{0}^{1} f(x) x^{2 \nu+1} P_{\nu}\left(\lambda_{n} x\right) d x,
$$

$\lambda_{n}$ being one of the positive roots of the transcendental equation $P_{v}(x)=0$;

where

$$
f(x)=(2 \nu+2) \int_{0}^{1} f(x) x^{2 \nu+1} d x+\sum_{1}^{\infty} p_{n}^{\prime} P_{\nu}\left(\lambda_{n}^{\prime} x\right)
$$

$$
p_{n}^{\prime}=\frac{2}{P_{\nu}^{2}\left(\lambda_{n}^{\prime}\right)} \int_{0}^{1} f(x) x^{2 v+1} P_{\nu}\left(\lambda_{n}^{\prime} x\right),
$$

$\lambda_{n}^{\prime}$ being one of the positive roots of the transcendental equation $P_{\nu}^{\prime}(x)=0$;

$$
f(x)=\sum_{1}^{\infty} p_{n}^{\prime \prime} P_{\nu}\left(\lambda_{n}^{\prime \prime} x\right)
$$

where

* These results in so far as they are independent of statements respecting uniform convergence may be found on pages 266, 267 of the Serie di Fourier of DINI, and I have reason to believe from a communication received from Professor DINI that the statements concerning uniform convergence have likewise been established by the same author, but remain as yet unpublished. 


$$
p_{n}^{\prime \prime}=\frac{2 \lambda_{n}^{\prime 2}}{\left\{h(2 \nu+h)+\lambda_{n}^{\prime 2}\right\} P_{\nu}^{2}\left(\lambda_{n}^{\prime \prime}\right)} \int_{0}^{1} f(x) x^{2 \nu+1} P_{\nu}\left(\lambda_{n}^{\prime \prime} x\right) d x,
$$

$\lambda_{n}^{\prime \prime}$ being one of the positive roots of the transcendental equation

$$
x P_{\nu}^{\prime}(x)-h P_{\nu}(x)=0, \quad(h \neq 0)
$$

provided throughout that $\nu>-\frac{1}{2}$ and that $f(x)$ satisfies the following conditions.

"Condition $(a): f(x)$ when considered in the interval $0 \leqq x \leqq 1$ is finite and either continuous or made up of a finite number of continuous portions.

"Condition $(b): f(x)$ possesses finite first derivatives from the right and from the left at the point $x$.

"Also, the above statement is true when $-1<\nu \leqq-\frac{1}{2}$ if in addition to these conditions we require that the function $\left|x^{2 v} f(x)\right|$ be integrable in the neighborhood at the right of the point $x=0$.

"Moreover, when $\nu>-\frac{1}{2}$ the series (2), (3) and (4) converge uniformly to the limit $f(x)$ when $a^{\prime}<x<b^{\prime}\left(0<a^{\prime}<b^{\prime}<1\right)$ provided that the function $f(x)$ when considered in the interval $0 \leqq x \leqq 1$ satisfies condition $(a)$, and when considered throughout the interval $a^{\prime} \leqq x \leqq b^{\prime}$ is continuous and possesses a finite first derivative from the right and from the left. And the same is true when $-1<\nu \leqq-\frac{1}{2}$ provided that in addition to these requirements the function $\left|x^{2 v} f(x)\right|$ is integrable in the neighborhood at the right of the point $x=0$."

2. This premised, we shall now assume that we are dealing with a function $f(x)$ which satisfies condition $(a)$, but instead of condition $(b)$ it satisfies the following two conditions which place somewhat further restrictions upon it:

Condition $(c): f(x)$ when considered within the interval $0<x<1$ possesses a continuous derivative $f^{\prime}(x)$ such that the function $\left|f^{\prime}(x)\right| / x$ when considered in the neighborhood of the point $x=0$ remains always less than a fixed constant $c$.

Condition $(d): f(x)$ possesses a finite second derivative from the right and from the left throughout the interval $a^{\prime} \leqq x \leqq b^{\prime}$.

Assuming then that $\nu>-1$ and that conditions $(a),(c)$ and $(d)$ are satisfied together with the condition when $-1<\nu \leqq-\frac{1}{2}$ that the functions $\left|x^{2 \nu} f(x)\right|$ and $\left|x^{2 \nu-1} f^{\prime}(x)\right|$ are integrable in the neighborhood at the right of the point $x=0$, it is evident that for any special value of $x$ such that $a^{\prime}<x<b^{\prime}$ condition $(b)$ becomes satisfied so that in particular we shall have (2) for such a value of $x$. And, if we admit for the moment the possibility of differentiating the series term by term, we have for the same value of $x$

$$
f^{\prime}(x)=\sum_{1}^{\infty} p_{n} P_{\nu}^{\prime}\left(\lambda_{n} x\right) \text {. }
$$

In order to justify (5) it suffices, as is well known,* to show that for the interval

*Vid. Osgood in American Journal of Mathematics, vol. 19, p. 155 et seq. 
$a^{\prime}<x<b^{\prime}$ the series in (5) is uniformly convergent and we shall now show that this is the case when $f(x)$ satisfies the conditions which we have supposed, together with one other, viz., $f(1)=0$. In passing, however, let us observe that from (1) we have

$$
P_{\nu}^{\prime}\left(\lambda_{n} x\right)=-\frac{\lambda_{n}^{2} x}{2 \nu+2} P_{\nu+1}\left(\lambda_{n} x\right)
$$

so that the series (5) may be written in the form

$$
-\sum_{1}^{\infty} \frac{p_{n} \lambda_{n}^{2} x}{2 \nu+2} P_{\nu+1}\left(\lambda_{n} x\right) .
$$

Now, utilizing the results stated at the beginning, we may write under the present hypothesis concerning $f^{\prime}(x)$

$$
\frac{f^{\prime}(x)}{x}=\sum_{1}^{\infty} p_{n}^{\prime \prime} P_{\nu+1}\left(\lambda_{n}^{\prime \prime} x\right)
$$

where

$$
p_{n}^{\prime \prime}=\frac{2 \lambda_{n}^{\prime \prime 2}}{\left\{h(2 \nu+2+h)+\lambda_{n}^{\prime \prime 2}\right\} P_{\nu+1}^{2}\left(\lambda_{n}^{\prime \prime}\right)} \int_{0}^{1} f^{\prime}(x) x^{2 v+2} P_{v+1}\left(\lambda_{n}^{\prime \prime} x\right) d x
$$

$\lambda_{n}^{\prime \prime}$ being one of the positive roots of the equation

$$
x P_{\nu+1}^{\prime}(x)-h P_{\nu+1}(x)=0,
$$

and from the results stated above we know that (8) holds uniformly when $a^{\prime}<x<b^{\prime}$. From (6) we have

$$
P_{\nu+1}^{\prime}(x)=-\frac{2 \nu+2}{x} P_{\nu}^{\prime \prime}(x)+\frac{2 \nu+2}{x^{2}} P_{\nu}^{\prime}(x),
$$

and hence (10) may be written

$$
-P_{\nu}^{\prime \prime}(x)+\frac{1+h}{x} P_{\nu}^{\prime}(x)=0
$$

so if we take $h=-2 \nu-2$ (which is consistent with $h \neq 0$ since $\nu>-1$ ) (10) reduces to

$$
-P_{\nu}^{\prime \prime}(x)-\frac{2 \nu+1}{x} P_{\nu}^{\prime}(x)=0 .
$$

But from (1) we have

$$
P_{\nu}^{\prime \prime}(x)+\frac{2 \nu+1}{x} P_{\nu}^{\prime}(x)+P_{\nu}(x)=0,
$$

and hence (12) is equivalent to the equation $P_{\nu}(x)=0$, so that having taken $h=-2 \nu-2$ we obtain a particular development of the form $(4)$ in which 
$\lambda_{n}^{\prime \prime}=\lambda_{n}$ and in which the coefficients $p_{n}^{\prime \prime}$ as given by (9) reduce to the more simple form

$$
p_{n}^{\prime \prime}=\frac{2}{P_{v+1}^{2}\left(\lambda_{n}\right)} \int_{0}^{1} f^{\prime}(x) x^{2 v+2} P_{v+1}\left(\lambda_{n} x\right) d x
$$

or again, utilizing (6), to

$$
p_{n}^{\prime \prime}=\frac{2 \lambda_{n}^{2}}{(2 \nu+2)^{2} P_{\nu}^{\prime 2}\left(\lambda_{n}\right)} \int_{0}^{1} f^{\prime}(x) x^{2 \nu+2} P_{\nu+1}\left(\lambda_{n} x\right) d x .
$$

In (13) let us now integrate once by parts, taking for this purpose

$$
d v=f^{\prime}(x) d x \quad \text { and } \quad u=x^{2 v+2} P_{v+1}\left(\lambda_{n} x\right) .
$$

Then $v=f(x)$ and noting that

$$
\frac{d}{d x}\left\{x^{2 \nu+2} P_{\nu+1}(x)\right\}=(2 \nu+2) x^{2 \nu+1} P_{\nu}(x)
$$

we have $d u=(2 \nu+2) x^{2 \nu+1} P_{\nu}\left(\lambda_{n} x\right) d x$, so that we may again write for $p_{n}^{\prime \prime}$

$$
\begin{aligned}
p_{n}^{\prime \prime} & =\frac{2 \lambda_{n}^{2}}{(2 \nu+2)^{2} P_{\nu}^{\prime 2}\left(\lambda_{n}\right)}\left[x^{2 \nu+1} f(x) P_{\nu+1}\left(\lambda_{n} x\right)\right]_{0}^{1} \\
& \quad-\frac{2 \lambda_{n}^{2}}{(2 \nu+2) P_{\nu}^{\prime 2}\left(\lambda_{n}\right)} \int_{0}^{1} f(x) x^{2 \nu+1} P_{\nu}\left(\lambda_{n} x\right) d x \\
= & \frac{2 \lambda_{n}^{2}}{(2 \nu+2)^{2} P_{\nu}^{\prime 2}\left(\lambda_{n}\right)}\left[x^{2 \nu+2} f(x) P_{\nu+1}\left(\lambda_{n} x\right)\right]_{0}^{1}-\frac{p_{n} \lambda_{n}^{2}}{2 \nu+2} .
\end{aligned}
$$

Therefore, since $\nu>-1$, we have but to assume that $f(1)=0$ in order to have the development (8) assume the form

$$
\frac{f^{\prime}(x)}{x}=-\sum_{1}^{\infty} \frac{p_{n} \lambda_{n}^{2}}{2 \nu+2} P_{\nu+1}\left(\lambda_{n} x\right) .
$$

Thus the series (7) is a special form of the $\mathrm{r}$ niformly convergent series (8) and is therefore itself uniformly convergent $\left(a^{\prime}<x<b^{\prime}\right)$.

Keeping the same hypotheses respecting $\nu$ and $f(x)$ we may show also that the series (3) when differentiated term by term will converge to the limit $f^{\prime}(x)$ when $a^{\prime}<x<b^{\prime}\left(0<a^{\prime}<b^{\prime}<1\right)$.

We have, in fact, upon differentiating both members of (3)

$$
f^{\prime}(x)=\sum_{1}^{\infty} p_{n}^{\prime} P_{\nu}^{\prime}\left(\lambda_{n}^{\prime} x\right)=-\sum_{1}^{\infty} \frac{p_{n}^{\prime} \lambda_{n}^{\prime 2} x}{2 \nu+2} P_{\nu+1}\left(\lambda_{n}^{\prime} x\right)
$$


where the last series may be shown as follows to converge uniformly for

$$
a^{\prime}<x<b^{\prime} .
$$

From (6) the positive roots $\lambda_{n}^{\prime}$ which appear in (14) and which by hypothesis are roots of $P_{\nu}^{\prime}(x)=0$ are the same as the positive roots of the equation $P_{v+1}(x)=0$, and hence by $(2)$ and the results stated at the beginning, we have uniformly when $a^{\prime}<x<b^{\prime}$

$$
\frac{f^{\prime}(x)}{x}=\sum_{1}^{\infty} p_{n} P_{v^{\prime+1}}\left(\lambda_{n}^{\prime} x\right)
$$

where

$$
p_{n}=\frac{2}{P_{\nu+1}^{\prime 2}\left(\lambda_{n}^{\prime}\right)} \int_{0}^{1} f^{\prime}(x) x^{2 v+2} P_{\nu+1}\left(\lambda_{n}^{\prime} x\right) d x
$$

But

and hence

$$
P_{\nu+1}(x)=(2 \nu+2) \frac{P_{\nu}^{\prime}(x)}{x},
$$

Therefore

$$
P_{\nu+1}^{\prime}(x)=(2 \nu+2) \frac{P_{\nu}^{\prime \prime}(x)}{x}-(2 \nu+2) \frac{P_{\nu}^{\prime}(x)}{x^{2}}
$$

or since in general

$$
P_{\nu+1}^{\prime}\left(\lambda_{n}^{\prime}\right)=(2 \nu+2) \frac{P_{\nu}^{\prime \prime}\left(\lambda_{n}^{\prime}\right)}{\lambda_{n}^{\prime}}
$$

$$
P_{\nu}^{\prime \prime}(x)+\frac{2 \nu+1}{x} P_{\nu}^{\prime}(x)+P_{\nu}(x)=0,
$$

we may use the fact that $P_{\nu}^{\prime \prime}\left(\lambda_{n}^{\prime}\right)=-P_{\nu}\left(\lambda_{n}^{\prime}\right)$ and write

$$
P_{\nu+1}^{\prime}\left(\lambda_{n}^{\prime}\right)=-(2 \nu+2) \frac{P_{\nu}^{\prime}\left(\lambda_{n}^{\prime}\right)}{\lambda_{n}^{\prime}} .
$$

Thus, formula (16) may be written

$$
p_{n}=\frac{2 \lambda_{n}^{\prime 2}}{(2 \nu+2)^{2} P_{\nu}^{2}\left(\lambda_{n}^{\prime}\right)} \int_{0}^{1} f^{\prime}(x) x^{2 \nu+2} P_{\nu+1}\left(\lambda_{n}^{\prime} x\right) d x
$$

and hence, with the present hypotheses concerning $f(x)$ we obtain, as in dealing with (13), the result that $p_{n}=-p_{n}^{\prime} \lambda_{n}^{\prime 2} /(2 \nu+2)$. Consequently the series (15) which we know is uniformly convergent for $a^{\prime}<x<b^{\prime}$ assumes the form

$$
\frac{f^{\prime}(x)}{x}=-\sum_{1}^{\infty} \frac{p_{n}^{\prime} \lambda_{n}^{\prime 2}}{2 \nu+2} P_{\nu+1}\left(\lambda_{n}^{\prime} x\right)
$$

from which the uniform convergence of the last series in (14) follows at once for the interval $a^{\prime}<x<b^{\prime}$. 
Introducing into the developments (2) and (3) the function $J_{\nu}(x)$ instead of $P_{\nu}(x)$, recalling that $J_{\nu}(x)=x^{\nu} P_{\nu}(x)$, and applying our results to the function $x^{-v} f(x)$ instead of $f(x)$ we obtain the following

Theorem: Each of the series I and II converges, when $a^{\prime}<x<b^{\prime}$ $\left(0<a^{\prime}<b^{\prime}<1\right)$, to the limit $f(x)$ and each of the series obtained by differentiating these series term by term converges for the same values of $x$ to the limit $f^{\prime \prime}(x)$, provided that $\nu>-\frac{1}{2}$ and that the function $\phi(x)=x^{-\nu} f(x)$ satisfies the following conditions:

Condition $A: \phi(x)$ when considered in the interval $0 \leqq x \leqq 1$ is finite and either continuous or made up of a finite number of continuous portions.

Condition $B: \phi(x)$ when considered in the interval $0<x<1$ possesses a continuous derivative $\phi^{\prime}(x)$ such that the function $\left|\phi^{\prime}(x)\right| / x$ when considered in the neighborhood of the point $x=0$ is less than a fixed constant.

Condition $C: \phi(x)$ when considered in the interval $a^{\prime} \equiv x \equiv b^{\prime}$ possesses finite second derivatives from the right and from the left.

Condition $D: \phi(1)=0$.

Moreover, when $-1>\nu \geqq-\frac{1}{2}$ the above theorem holds true if we require also that the functions $\left|x^{\nu} f(x)\right|$ and $\left|x^{\nu-1} f^{\prime}(x)\right|$ be integrable in the neighborhood at the right of the point $x=0$.

UNIVERSITY OF MICHIGAN, June, 1902. 